\title{
Correlation Between Cerebral Atrophy and Texture Features in Alzheimer-type Dementia Brains: A 3-Year Follow-up MRI Study
}

\author{
Naoki Kodama* Member \\ Hiroshi Takeuchi* ${ }^{*}$ Non-member
}

\begin{abstract}
We assessed relationships between six texture features and changes in atrophy of the cerebral parenchyma, the hippocampus, and the parahippocampal gyrus in the Alzheimer-type dementia (ATD) brain to determine whether or not the features reflect cerebral atrophy in ATD patients. The subjects of this study were 10 ATD patients, and underwent an magnetic resonanse imaging test of the head annually for at least 3 consecutive years. They consisted of three men and seven women, with a mean age of $71.4 \pm 6.7$ years. The results of study, the mean run length nonuniformity (RLN), angular second moment (ASM), and contrast $(\mathrm{CON})$ increased with time, whereas the mean gray level nonuniformity (GLN), run percentage (RPC), and entropy (ENT) decreased with time. There was a statistically significant correlation between brain-intracranial area ratio (BIR) and GLN ( $p=0.039)$, between BIR and ASM $(p=0.011)$, and between BIR and ENT $(p=0.023)$ as well as between parahippocampal-intracranial area ratio and GLN $(p=0.049)$. These results indicate that the six texture features were shown to reflect gray matter atrophy associated with ATD and to change with the progress of the disease. Although the course of ATD can be followed up by measuring a hippocampal area or volume and determining a decrease in the area or volume, texture features should be a more effective instrument for identifying the progress of ATD.
\end{abstract}

Keywords : Alzheimer-type dementia, texture features, magnetic resonance imaging, cerebral atrophy

\section{Introduction}

Many studies on the diagnosis of Alzheimer-type dementia (ATD) have been conducted with radiologic examinations, cognitive function rating scales (e.g., Mini-Mental State Examination [MMSE]), and other clinical instruments. The most common study theme is image-based diagnosis of ATD with magnetic resonance imaging (MRI) ${ }^{(1)-(6)}$. The determination of hippocampal area or volume with MRI has revealed that hippocampal atrophy is significantly more remarkable in ATD patients than in healthy controls.

The area or volume of the hippocampus and the parahippocampal gyrus has been determined on MRI manually by skilled neurologists or radiologists. Recently, many researchers have proposed automatic techniques for extracting the image of the hippocampus, which becomes atrophic specifically in ATD patients, from MRI. The techniques allow the objective image-based diagnosis of ATD. Hsu et al. reported no statistically significant difference in hippocampal volumes between automatic and manual techniques for extracting hippocampal information from $\mathrm{MRI}^{(7)}$. This indicates that the automatic hippocampal volumetry is a reliable technique.

We have studied and reported the image-based diagnosis of ATD with texture features ${ }^{(8)(9)}$. Quantitative texture feature measurements based on MRI allow not only numerical expression of morphological changes in the brain but also automatic computer calculations of the changes after an MRI slice for the calculations is selected manually. However, the correlation between cerebral atrophy and texture features in the ATD brain is poorly understood. We assessed relationships between six texture features and changes in atrophy of the cerebral

\footnotetext{
* Takasaki University of Health and Welfare.
}

37-1, Nakaohrui, Takasaki, 370-0033 parenchyma, the hippocampus, and the parahippocampal gyrus in the ATD brain to determine whether or not the features reflect cerebral atrophy in ATD patients.

\section{Subjects and Methods}

2.1 Subjects The subjects of this study were 10 ATD patients who visited Kawase Neurology Clinic (Niigata, Japan), were considered to have probable Alzheimer's disease according to NINCDS-ADRDA ${ }^{(10)}$, and underwent an MRI test of the head annually for at least 3 consecutive years and MMSE every 3 to 6 months. They consisted of three men and seven women, with a mean age of $71.4 \pm 6.7$ years. Written informed consent was obtained from all the patients and their family. The mean MMSE score was $17.3 \pm 5.4$ at baseline. A trained examiner performed MMSE to avoid score variabilities between examiners.

2.2 MRI Studies MRI studies of the head were conducted with a 0.2-T MRI unit (Magnetom P8 plus, Siemens). T1-weighted coronal images (three-dimensional; three-slab; repetition time, $50 \mathrm{msec}$; echo time, $12 \mathrm{msec}$; flip angle, $40^{\circ}$; field of view, $200 \mathrm{~mm}$; matrix, $240 \times 240$ ) were obtained with $3-\mathrm{mm}$ slices parallel to the anterior surface of brain stem. This plane was perpendicular to the long axis of hippocampus. When at least two slices included hippocampal information, a slice at the level of cerebral peduncle was used to measure hippocampal area.

2.3 Image Processing MRI processing procedures are illustrated in Figure 1. An original MRI of the head (Figure 2) was entered in a computer to perform binary operation. After the 4-nearest-neighbor erosion operation was performed twice, the image was labeled to extract information about the maximum parenchymal area. The 4-nearest-neighbor dilation operation was performed twice to extract an image of the cerebral parenchyma (Figure 3). Six texture features were measured quantitatively from the image as described below. 


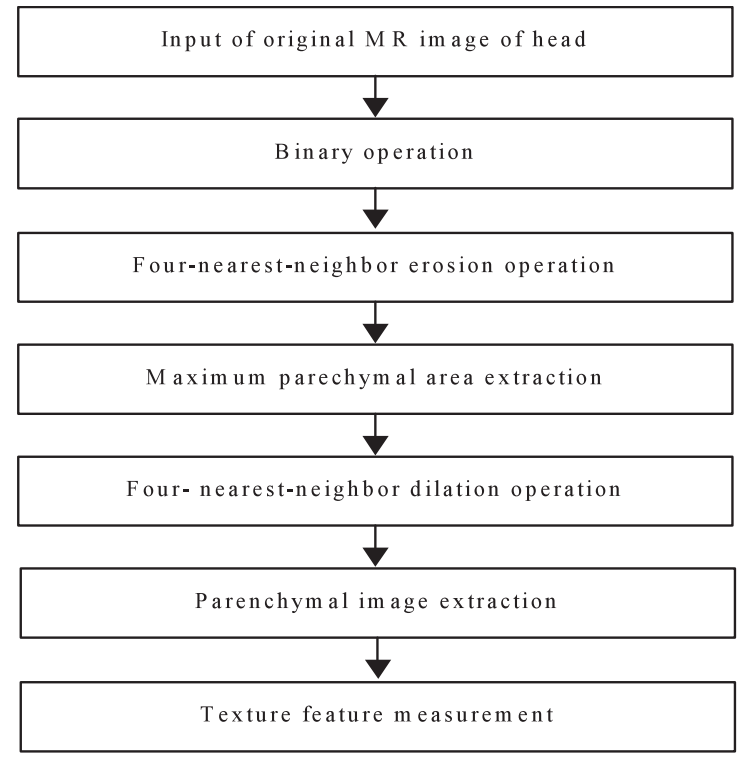

Fig. 1. Image processing procedures

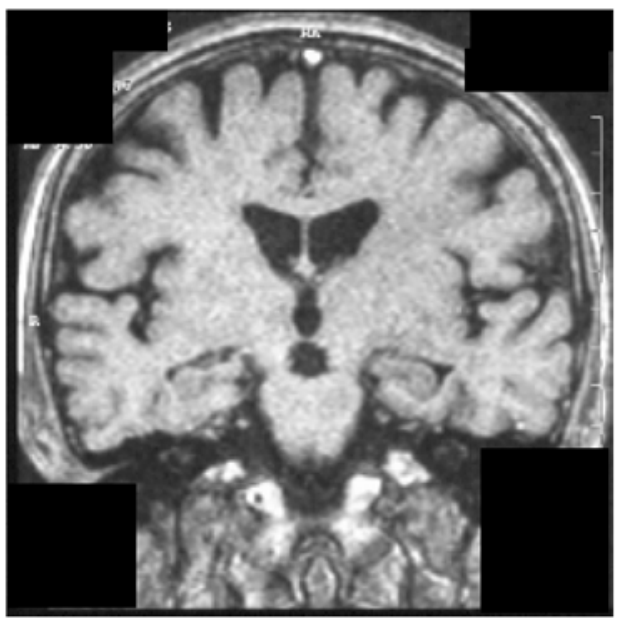

Fig. 2. Original MRI of the head

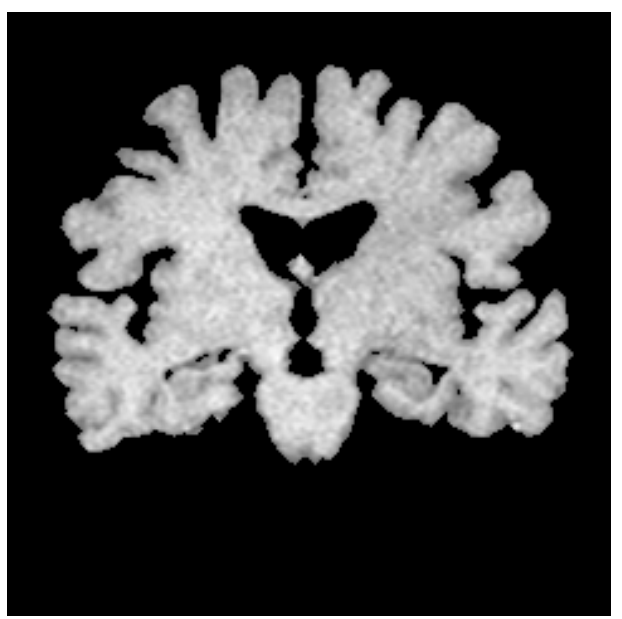

Fig. 3. Extracted parenchymal MRI

2.4 Brain-intracranial, Hippocampal-intracranial, and Parahippocampal-intracranial Area Ratios To assess relationships between hippocampal and parahippocampal atrophy and texture features, the brain-intracranial area ratio (BIR), the

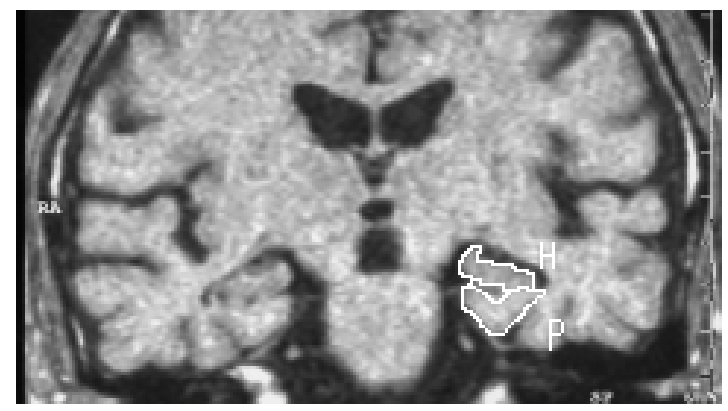

$\mathrm{H}=$ hippocampus, $\mathrm{P}=$ parahippocampal gyrus

Fig. 4. Definition of hippocampal and parahippocampal regions

hippocampal-intracranial area ratio (HIR), and the parahippocampal-intracranial area ratio (PIR) were calculated with the following equations:

$$
\begin{aligned}
& \mathrm{BIR}=\mathrm{B} / \mathrm{IC} \times 100 \\
& \mathrm{HIR}=\mathrm{H} / \mathrm{IC} \times 100 \\
& \mathrm{PIR}=\mathrm{P} / \mathrm{IC} \times 100 \cdots
\end{aligned}
$$

where $B$ is a brain area, $H$ is a hippocampal area, $P$ is a parahippocampal area, and $I C$ is an intracranial area. For these calculations, both a neurologist and a radiology technician defined the hippocampal and parahippocampal regions by tracing the structures visualized on the cathode ray tube (Fig. 4). They were not informed of the name, age, sex, diagnosis of the illness, or any other information of the patients for individual images.

The hippocampus is divided into three sections: an anterior segment (or head), a middle segment (or body), and a posterior segment (or tail). The term hippocampus refers to a constellation of anatomically distant structures, including the dentate gyrus, the Cornu Ammonis, the alveus hippocampi, and the fimbria hippocampi. The Cornu Ammonis is divided into CA1, CA2, CA3, and CA4 fields according to its cellular structures. The CA1 field is connected to the subiculum, the presubiculum, and the parahippocampal gyrus. The CA3 and CA4 fields are connected to the dentate gyrus. We defined the hippocampal region as CA1, CA2, CA3, and CA4 fields, the subiculum, the presubiculum, the dentate gyrus, and the fimbria hippocampi. The amygdaloid body was excluded from the region. To trace the hippocampal region accurately, horizontal and coronal MRIs were used as reference if necessary.

2.5 Run-length Matrix-based Texture Features The run-length matrix $P_{\theta}(i, j)$ represents the frequency that $j$ points with a gray level $i$ continue in the direction $\theta^{(11)(12)}$. The length of a run is referred to as run-length and expresses textures quantitatively. There are five run-length matrix-based texture features: short runs emphasis, long runs emphasis, gray level nonuniformity (GLN), run length nonuniformity (RLN), and run percentage (RPC). Of these features, we calculated GLN, RLN, and RPC because they were significantly different between ATD patients and healthy controls in our previous study ${ }^{(8)(9)}$. Prior to the calculation, each MRI was expressed in a 64-grayscale mode. When no minor variations in gray levels were taken into consideration for the calculation, more stable results were expected to be obtained.

$$
G L N=\sum_{i=0}^{n-1}\left\{\sum_{j=1}^{l} P_{\theta}(i, j)\right\}^{2} / \sum_{i=0}^{n-1} \sum_{j=1}^{l} P_{\theta}(i, j)
$$




$$
\begin{aligned}
& R L N=\sum_{j=1}^{i}\left\{\sum_{i=0}^{n-1} P_{\theta}(i, j)\right\}^{2} / \sum_{i=0}^{n-1} \sum_{j=1}^{l} P_{\theta}(i, j) \\
& R P C=\sum_{i=0}^{n-1} \sum_{j=1}^{l} P_{\theta}(i, j) / A
\end{aligned}
$$

where $A$ is an area, $\mathrm{i}$ and $\mathrm{n}$ are the number of gray levels, $\mathrm{j}$ and 1 are the run-length, and $P_{\theta}(i, j)$ is the run-length matrix of the detection $\theta$.

\subsection{Co-occurrence Matrix-based Texture Features}

The co-occurrence matrix $P_{\delta}(i, j)$ is the joint probability occurrence of gray levels $i$ and $j$ for two pixels with a defined spacial relationship $\delta=(r, \theta)^{(11)(12)}$. There are 14 co-occurrence matrix-based texture features. We calculated three of these features: angular second moment (ASM), contrast (CON), and entropy (ENT). They were significantly different between ATD patients and healthy controls in our previous study ${ }^{(8)(9)}$. Prior to the calculation, each MRI was expressed in a 64-grayscale mode, as with the calculation of the run-length matrix-based text features.

$$
\begin{aligned}
& P_{x-y}(k)=\sum_{i=0}^{n-1} \sum_{j=0}^{n-1} P_{\delta}(i, j) \\
& A S M=\sum_{i=0}^{n-1} \sum_{j=0}^{n-1}\left\{P_{\delta}(i, j)\right\}^{2} \\
& C O N=\sum_{k=0}^{n-1} k^{2} \cdot P_{x-y}(k) \\
& E N T=-\sum_{i=0}^{n-1} \sum_{j=0}^{n-1} P_{\delta}(i, j) \cdot \log \left\{P_{\delta}(i, j)\right\}
\end{aligned}
$$

where $\mathrm{n}$ is the number of gray levels, $\mathrm{i}$ and $\mathrm{j}$ are pixels, and $P_{\delta}(i, j)$ is the co-occurrence matrix.

\section{Results}

Table 1 shows changes in the mean BIR, HIR, PIR, and MMSE score over 3 years with the mean annualized percentage change (APC) for each parameter. All data in this report are presented as mean \pm standard deviation. The mean BIR was $66.58 \pm 2.30 \%$ at baseline and decreased by an average of $3.51 \%$ per year. The mean HIR was $1.57 \pm 0.42 \%$ at baseline and decreased by an average of $6.13 \%$ per year. The mean PIR was $1.80 \pm 0.31 \%$ at baseline and decreased by an average of $6.09 \%$ per year. The mean MMSE score was $17.3 \pm 5.40$ at baseline and decreased by approximately three points per year.

Table 2 summarizes changes in the mean run-length and co-occurrence matrix-based texture features over 3 years with the

Table 1. Changes in the mean BIR, HIR, PIR, and MMSE scores over 3 years

\begin{tabular}{|c|c|c|c|c|c|}
\hline & \multicolumn{4}{|c|}{ Time after initial visit (year) } & APC \\
\cline { 2 - 5 } & 0 & 1 & 2 & 3 & $(\%)$ \\
\hline & & & & & \\
BIR & $66.58 \pm 2.30$ & $64.3 \pm 3.17$ & $62.2 \pm 2.36$ & $59.9 \pm 3.50$ & -3.51 \\
HIR & $1.57 \pm 0.42)$ & $1.42 \pm 0.33$ & $1.38 \pm 0.37$ & $1.34 \pm 0.20$ & -6.13 \\
PIR & $1.80 \pm 0.31$ & $1.70 \pm 0.32$ & $1.59 \pm 0.32$ & $1.52 \pm 0.28$ & -6.09 \\
MMSE & $17.3 \pm 5.4$ & $13.7 \pm 6.7$ & $10.2 \pm 8.1$ & $9.0 \pm 7.1$ & -24.5 \\
& & & & & \\
\hline
\end{tabular}

$\mathrm{BIR}=$ brain-intracranial area ratio; $\mathrm{HAI}=$ hippocampal-intracranial area ratio; $\mathrm{PIR}=$ parahippocampal-intracranial area ratio; $\mathrm{MMSE}=$ Mini-Mental State Examination; $\mathrm{APC}=$ annualized percentage change.
Table 2. Changes in the mean texture features over 3 years

\begin{tabular}{|c|c|c|c|c|c|}
\hline \multirow{2}{*}{ Feature } & \multicolumn{4}{|c|}{ Time after initial visit (year) } & \multirow{2}{*}{$\begin{array}{c}\text { APC } \\
(\%)\end{array}$} \\
\cline { 2 - 5 } & 0 & 1 & 2 & 3 & -1.81 \\
\hline GLN & $890.9 \pm$ & $901.8 \pm$ & $849.9 \pm$ & $822.0 \pm$ & 163.1 \\
& 99.8 & 120.0 & 179.7 & $10691 \pm$ & 1.32 \\
RLN & $10404 \pm$ & $10491 \pm$ & $10640 \pm$ & 10691 \\
& 582 & 1167 & 1243 & 1407 & \\
RPC & $0.268 \pm$ & $0.268 \pm$ & $0.265 \pm$ & $0.259 \pm$ & -0.54 \\
& 0.008 & 0.018 & 0.019 & 0.020 & \\
ASM & $0.480 \pm$ & $0.482 \pm$ & $0.494 \pm$ & $0.505 \pm$ & 1.46 \\
& 0.015 & 0.024 & 0.028 & 0.019 & \\
CON & $26.05 \pm$ & $26.19 \pm$ & $26.14 \pm$ & $26.54 \pm$ & 0.41 \\
& 4.25 & 4.19 & 6.22 & 4.41 & \\
ENT & $2.784 \pm$ & $2.772 \pm$ & $2.730 \pm$ & $2.637 \pm$ & -0.96 \\
& 0.071 & 0.141 & 0.161 & 0.164 & \\
\hline
\end{tabular}

GLN = gray level nonuniformity; RLN = run length nonuniformity; RPC = run percentage; $\mathrm{ASM}=$ angular second moment; $\mathrm{CON}=$ contrast; $\mathrm{ENT}=$ entropy; $\mathrm{APC}=$ annualized percentage change

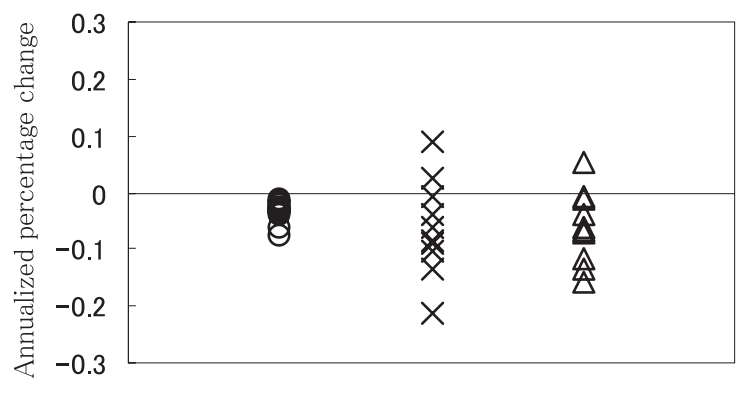

$\mathrm{BIR}=$ brain-intracranial area ratio $(\bigcirc)$; $\mathrm{HAI}=$ hippocampal-intracranial area ratio $(\times)$; $P I R=$ parahippocampal-intracranial area ratio. $(\triangle)$

Fig. 5. Annualized percentage change in three area ratios for 10 ATD patients

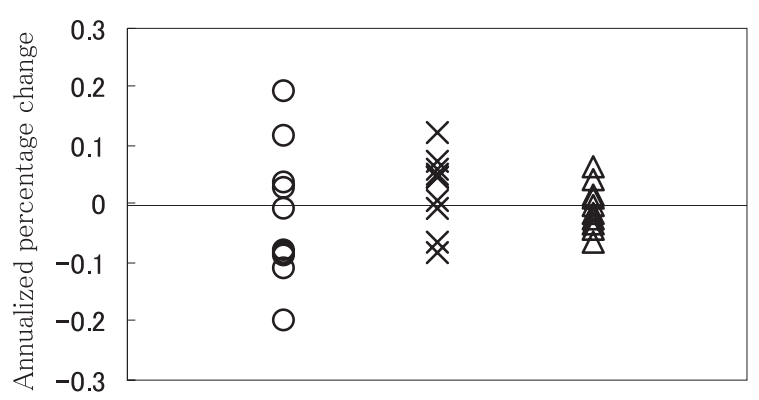

GLN $=$ gray level nonuniformity $(\bigcirc) ; \mathrm{RLN}=$ run length nonuniformity $(\times)$; RPC $=$ run percentage $(\triangle)$.

Fig. 6. Annualized percentage change in run-length matrix-based texture features for 10 ATD patients

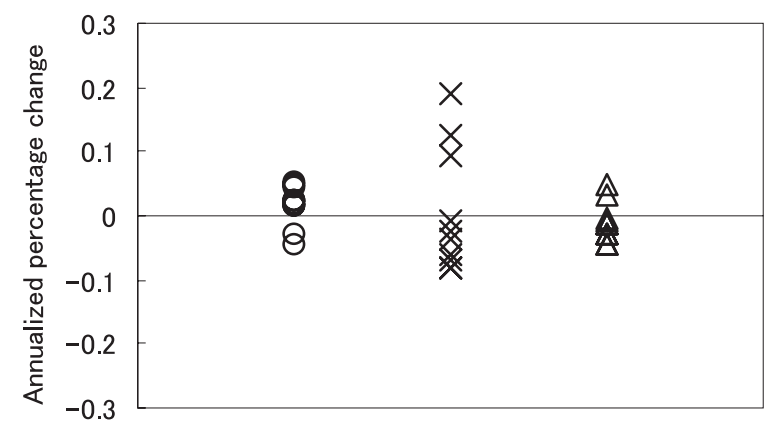

$\mathrm{ASM}=$ angular second moment $(\bigcirc) ; \mathrm{CON}=$ contrast $(\times)$; ENT $=$ entropy $(\triangle)$.

Fig. 7. Annualized percentage change in run-length matrix-based texture features for 10 ATD patients 
Table 3. Correlation coefficient for the relationship between three area ratios and six texture features

\begin{tabular}{|c|c|c|c|}
\hline & BIR & HIR & PIR \\
\hline GLN & $+0.685(0.039)^{*}$ & $+0.042(0.898)$ & $+0.612(0.049)^{*}$ \\
RLN & $-0.018(0.956)$ & $-0.103(0.753)$ & $-0.418(0.201)$ \\
RPC & $+0.382(0.252)$ & $+0.164(0.623)$ & $+0.042(0.897)$ \\
ASM & $-0.842(0.011)^{*}$ & $-0.333(0.317)$ & $-0.309(0.353)$ \\
CON & $-0.345(0.300)$ & $-0.079(0.813)$ & $-0.091(0.784)$ \\
ENT & $+0.758(0.023)^{*}$ & $+0.382(0.252)$ & $+0.188(0.573)$ \\
\hline
\end{tabular}

Data represent Spearman correlation coefficients for the relationship between

the annualized percentage change in the six texture features and the annualized percentage change in the three area ratios, with a $p$ value in parentheses. $* \mathrm{p}<0.05$

$\mathrm{BIR}=$ brain-intracranial area ratio; $\mathrm{HAI}=$ hippocampal-intracranial area ratio; $\mathrm{PIR}=$ parahippocampal-intracranial area ratio; GLN = gray level nonuniformity; RLN = run length nonuniformity; RPC = run percentage; ASM $=$ angular second moment $; \mathrm{CON}=$ contrast $; \mathrm{ENT}=$ entropy.

mean APC for each parameter. The mean RLN, ASM, and CON increased with time, whereas the mean GLN, RPC, and ENT decreased with time. The largest mean APC was $-1.81 \pm 1.15 \%$ for GLN, followed by $1.46 \pm 0.33 \%$ for ASM and $1.32 \pm 0.68 \%$ for RLN.

Figures 5, 6, and 7 present the APC in the three area ratios, the three run-length matrix-based texture features, and the three co-occurrence matrix-based texture features, respectively, for the individual patients. Table 3 shows the assessments of relationships between the APC in the three area ratios and the APC in the six texture features. There was a statistically significant correlation between BIR and GLN ( $p=0.039)$, between BIR and $\operatorname{ASM}(p=0.011)$, and between BIR and ENT $(p=0.023)$ as well as between PIR and GLN ( $p=0.049)$.

\section{Discussion}

Morphological changes in the ATD brain with time have recently been the focus of intense research interest. Jack et al. demonstrate that a hippocampal volume decreases at an average rate of $3.98 \pm 1.92 \%$ per year in ATD patients ${ }^{(13)}$. Laakso et al. report that right and left hippocampal volumes reduce by an average of $6.9 \pm 18.2 \%$ and $7.2 \pm 20.1 \%$, respectively, in a 3-year follow-up study ${ }^{(14)}$. In our study, the mean HIR and PIR reduced by $6.13 \pm 8.49 \%$ and $6.09 \pm 6.44 \%$ per year, respectively. These results suggest that hippocampal atrophy is similar in severity to the atrophy demonstrated by the above-mentioned studies, although we measured a hippocampal area whereas Jack et al. and Laakso et al. determined a hippocampal volume.

Many studies have been conducted on the image-based diagnosis of ATD. Du et al. manually measured volumes of the hippocampus and the entorhinal cortex based on MRI of the head. The rate of differentiation between ATD and normal cognition was $86 \%$ when hippocampal volumetry alone was performed; it was $89 \%$ when volumes of both the hippocampus and the entorhinal cortex were measured ${ }^{(15)}$. Wolf et al. performed hippocampal volumetry manually with a statistically significant difference in the right and left hippocampal volumes between ATD patients and healthy controls ${ }^{(16)}$. Mizuno et al. determined volumes of the hippocampus and the amygdaloid body with a statistically significant difference in the volumes between ATD patients and healthy controls ${ }^{(17)}$. Rusinek et al. automatically subtracted a difference between a baseline MRI of the head and a follow-up
MRI to calculate the temporal lobe atrophy rate for the diagnosis of ATD ${ }^{(18)}$. Most of such studies aim to evaluate the efficacy of techniques for measuring hippocampal volume or area in the diagnosis of ATD. The objective of our study was to determine whether or not texture features reflect cerebral atrophy in ATD patients, rather than whether or not an automatic technique for measuring hippocampal area with texture features is effective for the diagnosis of ATD.

Texture features can express morphological changes numerically. The applications of the features to medical images include a mammogram computer-aided diagnosis system to reduce false-positive candidates for nodular densities ${ }^{(19)}$, run-length analyses used in computer analyses of interstitial pneumonia ${ }^{(20)}$, and texture analyses of ultrasonographs ${ }^{(21)}$. The determination of texture features seems to allow the quantitative assessment of morphological changes in the brain associated with cerebral atrophy in ATD patients.

We analyzed six texture features that were significantly different between ATD patients and healthy controls in our previous study: GLN, RLN, RPC, ASM, CON, and ENT. In run-length matrix-based features, GLN is high if gray levels are not equal throughout an image. RLN is high if run-lengths are not equal throughout an image. RPC is high if many runs are present in a given image size. In co-occurrence matrix-based features, ASM expresses the presence of many specific pixel pairs. CON represents contrasts or local changes in an image. ENT indicates texture homogeneity.

Diffuse cerebral atrophy in ATD patients is characterized by atrophy of the gray matter including the hippocampus and the parahippocampal gyrus. With the progress of the disease, gray matter atrophy becomes remarkable. This should change the six texture features based on the following hypothesis. The relative area of the white matter increases as gray matter atrophy becomes severe. This can increase RLN and reduce GLN and RPC. Since gray matter atrophy results in relatively enlarged pixel pairs for the white matter as well as reduced uniformity of pixels, ASM is expected to be high whereas ENT is expected to be low with the progress of ATD. CON should elevate because gray matter atrophy increases local contrasts on an image.

We assessed relationships between the APC in the three area ratios (i.e., BIR, HIR, and PIR) and the APC in the six texture features. There was a statistically significant correlation between BIR and GLN ( $p=0.039)$, between BIR and ASM $(p=0.011)$, and between BIR and ENT $(p=0.023)$ as well as between PIR and GLN ( $p=0.049)$. No significant correlation was found between HIR and the six texture features. These results are explained probably by the calculation of the features based on extracted parenchymal MRI. If the six texture features are calculated based on the automatically extracted image of the hippocampus or parahippocampal gyrus, a strong correlation will be found between HIR or PIR and each feature. Further work is needed to assess relationships between hippocampal and parahippocampal gyrus volumes and the texture features.

Since there was a significant correlation between BIR and GLN, ASM, or ENT, these texture features were shown to reflect gray matter atrophy associated with ATD and to change with the progress of the disease. Although the course of ATD can be followed up by measuring a hippocampal area or volume and determining a decrease in the area or volume, texture features should be a more effective instrument for identifying the progress 
of ATD. The calculation of the features is easier and faster than the determination of hippocampal area or volume. A series of calculation procedures can be fully automated with a computer to offer results in several seconds. Once a physician or radiology technician selects an MRI slice of the head for the calculation, no further manual work is needed.

Continuing studies should be conducted with a larger sample size and assess relationships between each texture feature and the atrophy of the hippocampus and the parahippocampal gyrus based on extracted hippocampal and parahippocampal MRI.

\section{Conclusion}

We assessed relationships between six texture features and changes in atrophy of the cerebral parenchyma, the hippocampus, and the parahippocampal gyrus in the ATD brain to determine whether or not the features reflect cerebral atrophy in ATD patients. The following results were obtained:

(1) The mean BIR, HIR, and PIR decreased by an average of $3.51 \%, 6.13 \%$, and $6.09 \%$ per year, respectively. The mean MMSE score decreased by an average of approximately three points per year.

(2) The analysis of time course of six texture features revealed that the mean RLN, ASM, and CON increased whereas the mean GLN, RPC, and ENT decreased with the progress of ATD.

(3) A statistically significant correlation was found between the APC in BIR and the APC in GLN, ASM, or ENT.

(4) The six texture features were shown to reflect gray matter atrophy associated with ATD and to change with the progress of the disease.

(Manuscript received Dec. 20, 2004, revised June 16, 2005)

\section{References}

(1) M. P. Laakso, H. Soininen, K. Partanen, M. Lehtovirta, M. Hallikainen, T. Hanninen, E. L. Helkala, P. Vainio, and P. J. Riekkinen: "MRI of the hippocampus in Alzheimer's disease: sensitivity, specificity, and analysis of the incorrectly classified subjects", Neurobiol. Aging, Vol.19, No.1, pp.23-31 (1998)

(2) M. Golebiowski, M. Barcikowska, and A. Pfeffer: "Magnetic resonance imaging - based hippocampal volumetry in patients with dementia of the Alzheimer type", Dementia, Vol.10, No.4, pp.284-288 (1999)

(3) M. P. Laakso, G. B. Frisoni, M. Kononen, M. Mikkonen, A. Beltramello, C. Geroldi, A. Bianchetti, M. Trabucchi, H. Soininen, and H. J. Aronen: "Hippocampus and entorhinal cortex in frontotemporal dementia and Alzheimer's disease: a morphometric MRI study", Biol. Psychiatry, Vol.47, No.12, pp.1056-1063 (2000)

(4) R. J. Killiany, B. T. Hyman, T. Gomez-Isla, M. B. Moss, R. Kikinis, F. Jolesz, R. Tanzi, K. Jones, and M. S. Albert: "MRI measures of entorhinal cortex vs hippocampus in preclinical AD", Neurology, Vol.58, No.8, pp.1188-1196 (2002)

(5) K. Kantarci and C. R. Jack: "Neuroimaging in Alzheimer disease: an evidence-based review", Neuroimag. Clin. N. Am., Vol.13, No.2, pp.197-209 (2003)

(6) Y. Y. Hsu, N. Schuff, A. T. Du, K. Mark, X. Zhu, D. Hardin, and M. W. Weiner: "Comparison of automated and manual MRI volumetry of hippocampus in normal aging and dementia", J. Magn. Reson. Imaging, Vol.16, No.3, pp.305-310 (2002)

(7) C. R. Jack, M. M. Shiung, J. L. Cunter, P. C. O'Brien, S. D. Weigand, D. S. Knopman, B. F. Boeve, R. J. Ivnik, G. E. Smith, R. H. Cha, E. G. Tangalos, and R. C. Petersen: "Comparison of different MRI brain atrophy rate measures with clinical disease progression in AD", Neurology, Vol.62, No.4, pp.591-600 (2004)

(8) N. Kodama, T. Shimada, and I. Fukumoto: "Image-based diagnosis of Alzheimer-type dementia: Measurements of hippocampal and ventricular areas in MR images", Magnetic Resonance in Medical Sciences, Vol.1, No.1, pp.14-20 (2002)

(9) N. Kodama, T. Shimada, T. Kaeriyama, T. Kaneko, Y Kobayashi, and I. Fukumoto: "Application of textural features to the objective diagnosis of Alzheimer-type dementia", Med. Imag. Tech., Vol.21, No.1, pp.41-48 (2003)

(10) G. McKhann, D. Drachman, and M. Folstein: "Clinical diagnosis of Alzheimer's disease: report of the NINCDS-ADRDA work group under the auspices of department of health and human services task force on Alzheimer's disease", Neurology, Vol.34, No.7, pp.939-944 (1984)

(11) M. Takagi and H. Shimoda: Handbook of image analysis, University of Tokyo Press, Tokyo (1991)

(12) H. Shimoda: Image Processing, Computer Graphic Arts Society, Tokyo (1997)

(13) C. R. Jack, R. C. Petersen, Y. Xu, P. C. O'Brien, G. E. Smith, R. J. Ivnik, E. G. Tangalos, and E. Kokmen: "Rate of medial temporal lobe atrophy in typical aging and Alzheimer's disease", Neurology, Vol.51, No.4, pp.993-999 (1998)

(14) M. P. Laakso, M. Lehtovirta, K. Partanen, P. J. Riekkinen, and H. Soininen: "Hippocampus in Alzheimer's disease: A 3-year follow-up study", Biol. Psychiatry, Vol.47, No.12, pp.557-561 (2000)

(15) A. T. Du, N. Schuff, D. Amend, M. P. Laakso, Y. Y. Hsu, W. J. Jagust, K. Yaffe, J. H. Kramer, B. Reed, D. Norman, H. C. Chui, and M. W. Weiner: "Magnetic resonance imaging of the entorhinal cortex and hippocampus in mild cognitive impairment and Alzheimer's disease", J. Neurol. Neurosurg. Psychiatry, Vol.71, No.4, pp.441-447 (2001)

(16) H. Wolf, M. Grunwald, F. Kruggel, S. G. Riedel-Heller, S. Angerhofer, A. Hojjatoleslami, A. Hensel, T. Arendt, and H. Gertz: "Hippocampal volume discriminates between normal cognition; questionable and mild dementia in the elderly", Neurobiol. Aging, Vol.22, No.2, pp.177-186 (2001)

(17) K. Mizuno, M. Wakui, A. Takeda, and G. Sobue: "Medial temporal atrophy and memory impairment in early stage of Alzheimer's disease: an MRI volumetric and memory assessment study", J. Neurol. Sci., Vol.173, No.1, pp.18-24 (2000)

(18) H. Rusinek, S. De Santi, D. Frid, W. H. Tsui, C. Y. Tarshish, A. Convit, M. J. de Leon: "Regional brain atrophy rate predicts future cognitive decline: 6-year longitudinal MR imaging study of normal aging", Radiology, Vol.229, pp.691-696 (2003)

(19) O. Ohtsuka, S. Kasai, Y. Hatanaka, H. Fujita, T. Hara, and T. Endo: "Elimination of false-positive mass candidates using second-order statistics in a mammogram CAD system", Japan Society of Med. Imag. and Inf. Sci., Vol.16, No.1, pp.13-19 (1999)

(20) J. Morishita, S. Katsuragawa, and D. Kunio,:"Application of gray-level run length analysis of computerized analysis of interstitial on chest radiograph", Japanese Society of Radiological Technology, Vol.56, No.3, pp.384-390 (2000)

(21) Y. Wang, K. Itoh, N. Taniguchi, H. Toei, F. Kawai, M. Nakamura, K. Omoto, K. Yokota, and T. Ono: "Studies on tissue characterization by texture analysis with co-occurrence matrix method using ultrasonography and CT imaging", J. Med. Ultrasonics, Vol.26, No.1, pp.825-837 (1999)

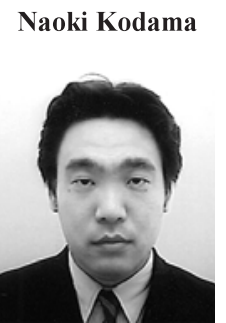

(Member) received Ph.D. degree from Nagaoka University of Technology, Japan. In 2004 he is currently a Research Associate in the Department of Healthcare Infomatics, at Takasaki University of Health and Welfare, Japan. His research interests include medical imaging.

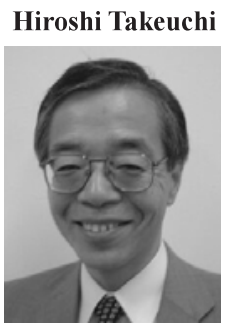

(Non-member) received Ph.D. degree from Waseda University, Japan. From 1971 to 2002, he was working in Central Reserch Laboratory, at Hitachi Ltd., Japan. He is currently a Professor in the Department of Healthcare Infomatics, at Takasaki University of Health and Welfare, Japan. His research interests included healthcare informatics for preventive medicine. 